Physics

Physics Research Publications

Simple waves in relativistic fluids

M. Lyutikov 


\title{
Simple waves in relativistic fluids
}

\author{
Maxim Lyutikov \\ Department of Physics, Purdue University, 525 Northwestern Avenue, West Lafayette, Indiana 47907-2036, USA
}

(Received 14 April 2010; published 9 November 2010)

\begin{abstract}
We consider the Riemann problem for relativistic flows of polytropic fluids and find relations for the flow characteristics. Evolution of physical quantities takes especially simple form for the case of cold magnetized plasmas. We find exact explicit analytical solutions for one-dimensional expansion of magnetized plasma into vacuum, valid for arbitrary magnetization. We also consider expansion into cold unmagnetized external medium both for stationary initial conditions and for initially moving plasma, as well as reflection of rarefaction wave from a wall. We also find self-similar structure of three-dimensional magnetized outflows into vacuum, valid close to the plasma-vacuum interface.
\end{abstract}

DOI: 10.1103/PhysRevE.82.056305

PACS number(s): 47.75.+f, 47.45.-n, 52.27.Ny, 52.35.Tc

\section{INTRODUCTION}

Relativistic shock waves are common in different physical systems [1], from heavy-ion nuclear collision (e.g., [2]) to astrophysical shocks in pulsar winds [3], active galactic nuclei (e.g., [4]), and gamma-ray bursts [5]. Many modern computational algorithms are based on the solution of the Riemann problems (e.g., [6]). These algorithms are based on Godunov-type shock-capturing schemes and do not require large artificial viscosity or smoothing operators. Analytical solutions to the corresponding Riemann problems are then important for code testing.

Exact explicit nonlinear solutions of relativistic fluid equations and especially relativistic magnetohydrodynamics equations are rare. In a general form the relativistic Riemann problem was solved by Martí and co-workers [7,8], who found the solutions for the Riemann invariants and for the characteristics in quadratures. In this paper we find simple expressions for the characteristics of the Riemann simple waves and in particular for the astrophysical important case of dynamics of a cold relativistically magnetized plasma. Our results can be used for benchmark estimates of the overall dynamical behavior in numerical simulations of relativistic flows and strongly magnetized outflows in particular.

\section{ONE-DIMENSIONAL EXPANSION OF POLYTROPIC GAS INTO VACUUM}

\section{A. Polytropic equations of state}

Let us assume that pressure is a polytropic function of density, $P=K \rho^{\Gamma}$, with constant $\Gamma$; then, the internal energy density, excluding rest mass, is $\mathcal{E}=P /(\Gamma-1)$, enthalpy $h=\rho$ $+\mathcal{E}+P=\rho+\Gamma /(\Gamma-1) P$, and sound speed can be expressed as

$$
c_{s}^{2}=\frac{\rho}{h} \frac{\partial P}{\partial \rho}=\frac{(\Gamma-1) \Gamma P / \rho}{(\Gamma-1)+\Gamma P / \rho} .
$$

The corresponding four-velocity is

$$
u_{s}^{2}=\frac{c_{s}^{2}}{1-c_{s}^{2}}=\frac{(\Gamma-1) \Gamma P / \rho}{(\Gamma-1)+\Gamma(2-\Gamma) P / \rho} .
$$

\section{B. Riemann invariants}

Consider a one-dimensional (1D) flow of fluid along $z$ direction, neglecting variations of quantities across the flow. The governing equations are

$$
\begin{gathered}
\partial_{t}(\gamma \rho)+\partial_{z}(\gamma \beta \rho)=0, \\
\partial_{t} T_{00}+\partial_{z} T_{0 z}=0, \\
\partial_{t} T_{0 z}+\partial_{z} T_{z z}=0, \\
T_{00}=\gamma^{2}(\mathcal{E}+P+\rho)-P, \\
T_{0 z}=\gamma^{2} \beta(\mathcal{E}+P+\rho), \\
T_{z z}=\gamma^{2} \beta^{2}(\mathcal{E}+P+\rho)+P,
\end{gathered}
$$

where $P$ is pressure, $\mathcal{E}$ is energy density (excluding rest mass), $\rho$ is density, and $\beta$ and $\gamma$ are fluid's velocities and Lorentz factors.

Assume that initially the fluid occupies region $z<0$ with constant density and pressure and that expansion proceeds into positive $z$ direction (Fig. 1).

Combining the conservation of energy and momentum, we find $[9,10]$

$$
\begin{gathered}
\left(\partial_{t}+\beta \partial_{z}\right) \beta=-\frac{\left(\beta \partial_{t}+\partial_{z}\right) P}{(\mathcal{E}+\rho+P) \gamma^{2}}, \\
\left(\partial_{t}+\beta \partial_{z}\right)(\mathcal{E}+\rho)=-(\mathcal{E}+\rho+P) \gamma^{2}\left(\beta \partial_{t}+\partial_{z}\right) \beta .
\end{gathered}
$$

These two equations and the equation of state combine to give equations for the Riemann invariants,

$$
\begin{aligned}
& \left(\partial_{t}+\frac{\beta+\beta_{c_{s}}}{1+\beta_{c_{s}} \beta} \partial_{z}\right) J_{+}=0, \\
& \left(\partial_{t}+\frac{\beta-\beta_{c_{s}}}{1-\beta_{c_{s}} \beta} \partial_{z}\right) J_{-}=0,
\end{aligned}
$$




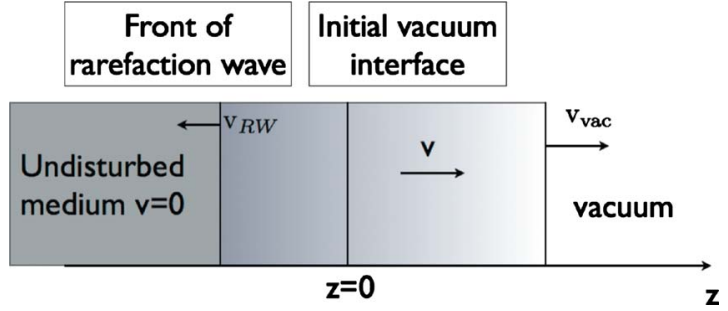

FIG. 1. (Color online) Geometry of the flow. Initially the fluid at rest occupies region $z<0$ with constant density and pressure and that expansion proceeds into positive $z$ direction. The fluid moves in the positive $z$ direction while the front of the rarefaction wave propagates in the negative $z$ direction.

$$
J_{ \pm}=\frac{1}{2} \ln \frac{1+\beta}{1-\beta} \pm \frac{1}{\sqrt{\Gamma-1}} \ln \frac{\sqrt{\Gamma-1}+c_{s}}{\sqrt{\Gamma-1}-c_{s}}
$$

[7]. ( $J_{+}$corresponds to the forward characteristic.) The combinations $\left(\beta \pm \beta_{c_{s}}\right) /\left(1 \pm \beta_{c_{s}} \beta\right)$ can be recognized as relativistic transformation of velocities, plasma velocity and wave phase velocity. The Riemann invariants are constant along characteristics

$$
d_{t} z_{ \pm}=\frac{\beta \pm \beta_{c_{s}}}{1 \pm \beta \beta_{c_{s}}}
$$

(the forward characteristics correspond to upper sign).

Equations (5) simplify if we introduce the Doppler factor

$$
\begin{gathered}
\delta_{\beta}=\frac{1}{(1-\beta) \gamma}=\sqrt{\frac{1+\beta}{1-\beta}}, \\
\delta_{\eta}=\sqrt{\frac{1+\eta}{1-\eta}}, \\
\delta_{c_{s}}=\sqrt{\frac{1+c_{s}}{1-c_{s}}} .
\end{gathered}
$$

(Under colinear Lorentz transformation, the corresponding Doppler factors change as $\delta \rightarrow \delta^{\prime} \delta_{\text {boost }}^{ \pm 1}$, where $\delta^{\prime}$ is a Doppler factor in a frame moving with respect to the observer frame with Doppler factor $\delta_{\text {boost }}$. Doppler factors multiply for aligned velocities or divide in case of counteraligned velocities.)

Next we assume that all quantities depend on self-similar coordinate $\eta=z / t$. When expressed in terms of Doppler factors, Eqs. (5) then simplify

$$
\begin{aligned}
& \left(\delta_{c_{s}}^{2} \delta_{\beta}^{2}-\delta_{\eta}^{2}\right) \partial_{\eta} J_{+}=0, \\
& \left(\delta_{\beta}^{2}-\delta_{\eta}^{2} \delta_{c_{s}}^{2}\right) \partial_{\eta} J_{-}=0 .
\end{aligned}
$$

This gives $\delta_{\beta}=\delta_{\eta} \delta_{c_{s}}$ for the forward and $\delta_{\beta}=\delta_{\eta} / \delta_{c_{s}}$ for the backward characteristics correspondingly.

Equation (9) is identically satisfied on the forward characteristics, while Eq. (8) is satisfied on the backward characteristics. Using the expressions for the Riemann invariants and the shape of characteristics we find

$$
\begin{gathered}
\partial_{\eta}\left[J_{+}\left(\delta_{\beta}=\delta_{c_{s}} \delta_{\eta}\right)\right]=0, \\
\partial_{\eta}\left(J_{-}\left(\delta_{\beta}=\delta_{\eta} / \delta_{c_{s}}\right)\right)=0,
\end{gathered}
$$

which give the full solution for simple waves in relativistic fluids,

$$
\begin{gathered}
\delta_{\eta,+}=\frac{C_{0}}{\delta_{c_{s}}}\left(\frac{1-c_{s} / \sqrt{\Gamma-1}}{1+c_{s} / \sqrt{\Gamma-1}}\right)^{1 / \sqrt{\Gamma-1}}, \\
\delta_{\beta}=\delta_{c_{s}} \delta_{\eta}, \text { forward characteristics, } \\
\delta_{\eta,-}=C_{0} \delta_{c_{s}}\left(\frac{1+c_{s} / \sqrt{\Gamma-1}}{1-c_{s} / \sqrt{\Gamma-1}}\right)^{1 / \sqrt{\Gamma-1}}, \\
\delta_{\beta}=\delta_{\eta} / \delta_{c_{s}}, \text { backward characteristics, }
\end{gathered}
$$

where $C_{0}$ is a constant to be determined from initial condition. Relations (12) give a general relation between the selfsimilar coordinate $\eta$, local sound speed in the flow $c_{s}$, and velocity of the flow $\beta$. These are transcendental equations for $c_{s}(\eta)$ and $\beta(\eta)$.

\section{Magnetized cold plasma}

In many astrophysical phenomena the magnetic field controls the overall dynamics of plasma. These likely include magnetars (strongly magnetized neutron stars possessing superstrong magnetic fields), pulsars and pulsar wind nebulae, jets of active galactic nuclei, and gamma-ray bursters. The plasma of these exotic objects can be described as relativistically strongly magnetized. This means that the inertia of this plasma is dominated by the magnetic field and not by the particle rest mass, $B^{2} / 8 \pi \gg \rho c^{2}$, and that the propagation speed of the Alfvén waves approaches the speed of light. Thus, the conditions in such plasmas are very different from the conditions encountered in the laboratory plasmas, the plasmas of planetary magnetospheres, and the interplanetary plasma.

For example, magnetic fields may play an important dynamical role in the gamma-ray burst outflows (e.g., [11,12]). They may power the relativistic outflow through, e.g., the Blandford-Znajek [13] process (see also [14]) and contribute to particle acceleration in the emission regions. The extreme physical conditions of magnetically dominated plasma suggest new physics and demand a systematic study. In relativistic magnetoplasma the Godunov-type schemes are discussed in [15].

Consider cold ideal magnetized plasma moving perpendicular to the direction of the magnetic field. This case, in fact, is just a particular case of polytropic equations of state $P \propto \rho^{\Gamma}$ for unmagnetized fluid flow [16]. The case of cold magnetized plasma is, in fact, somewhat special since it gives simple relations between density and sound fourvelocity, as we demonstrate below. For cold plasma, when the pressure is purely magnetic, $P=B^{2} / 2$ (for convenience, we renormalize magnetic field by $\sqrt{4 \pi}$ below) and polytropic index $\Gamma=2$, and defining magnetization parameter $\sigma=B^{2} / \rho$, Eq. (2) gives 


$$
U_{s} \equiv U_{A}=\frac{\beta_{A}}{\sqrt{1-\beta_{A}^{2}}}=\sqrt{\sigma},
$$

the four-velocity of the Alfvén and fast magnetosonic waves in strongly magnetized plasma [3] $\left(\beta_{A}\right.$ is the Alfvén velocity).

In case of magnetized plasma, the equations of motion (3) should be supplemented with induction equation and definitions of magnetic energy density and pressure,

$$
\begin{gathered}
\partial_{t}(\gamma B)+\partial_{z}(\gamma \beta B)=0, \\
\mathcal{E}=P=B^{2} / 2,
\end{gathered}
$$

where $B$ is a proper magnetic field. From Eqs. (14), it immediately follows that magnetic field and density are proportional, $B=\left(\rho / \rho_{0}\right) B_{0}$, where $\rho_{0}$ and $B_{0}$ are constants taken to be density and magnetic field in the initial state.

For $\Gamma=2$, the Riemann invariants take the form

$$
\begin{aligned}
& J_{+}=\ln \left(\delta_{\beta} \delta_{A}^{2}\right), \\
& J_{-}=\ln \left(\delta_{\beta} \delta_{A}^{-2}\right),
\end{aligned}
$$

where we introduced Doppler factors $\delta_{A}$,

$$
\delta_{A}=\frac{1}{\left(1-\beta_{A}\right) \gamma_{A}}=\sqrt{\frac{1+\beta_{A}}{1-\beta_{A}}},
$$

where $\beta_{A}$ is the local Alfvén speed.

General solution (12) then gives

$$
\delta_{A}=C_{0} \delta_{\eta}^{\mp 1 / 3}, \quad \delta_{\beta}=\delta_{\eta} \delta_{A}^{ \pm 1}=C_{0} \delta_{\eta}^{2 / 3},
$$

where $C_{0}$ is a constant to be determined from initial condition. Upper signs in Eqs. (17) correspond to the forward characteristics.

\section{Stationary initial conditions}

Assuming that initially the plasma is at rest, so that at the front of the rarefaction wave (RW) (where $\beta=0, \delta_{\beta}=1$ ) the Alfvén velocity corresponds to the Alfvén velocity in the unperturbed medium, given by corresponding Doppler factor $\delta_{A, 0}$, we find a simple fully analytical solution to the problem of one-dimensional relativistic expansion of magnetized gas into vacuum (see Fig. 2),

$$
\delta_{\beta}=\delta_{\eta}^{2 / 3} \delta_{A, 0}^{2 / 3}, \quad \delta_{A}=\frac{\delta_{A, 0}^{2 / 3}}{\delta_{\eta}^{1 / 3}} .
$$

The other solution, corresponding to expansion to the left, is

$$
\delta_{\beta}=\frac{\delta_{\eta}^{2 / 3}}{\delta_{A, 0}^{2 / 3}}, \quad \delta_{A}=\delta_{A, 0}^{2 / 3} \delta_{\eta}^{1 / 3} .
$$

These solutions give the velocity and magnetic field as functions of the self-similar coordinate $\eta$ and initial magnetization $\sigma$.

The relations for the more commonly used parameters are (for the forward characteristics)

$$
\gamma=\frac{1}{2}\left(\delta_{\eta}^{2 / 3} \delta_{A, 0}^{2 / 3}+\frac{1}{\delta_{\eta}^{2 / 3} \delta_{A, 0}^{2 / 3}}\right),
$$

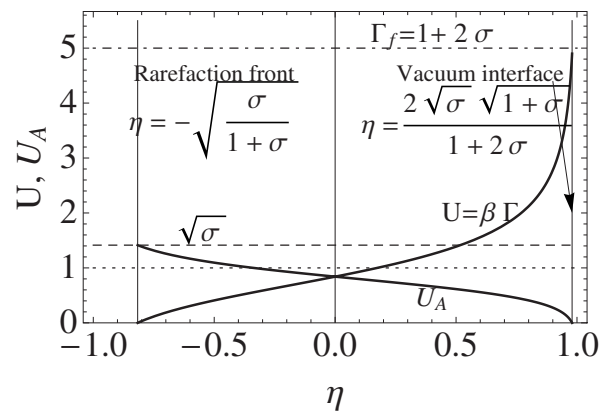

FIG. 2. Four-velocity of the flow and Alfvén four-velocity $U_{A}$ for one-dimensional self-similar expansion of magnetized gas into vacuum. For this plot $\sigma=2$.

$$
\begin{gathered}
\beta=\frac{\delta_{A, 0}^{4 / 3} \delta_{\eta}^{4 / 3}-1}{1+\delta_{A, 0}^{4 / 3} \delta_{\eta}^{4 / 3}}, \\
U_{A}=\frac{1}{2}\left(\frac{\delta_{A, 0}^{2 / 3}}{\delta_{\eta}^{1 / 3}}-\frac{\delta_{\eta}^{1 / 3}}{\delta_{A, 0}^{2 / 3}}\right), \\
B=\left(\frac{\delta_{A, 0}^{2 / 3}}{\delta_{\eta}^{1 / 3}}-\frac{\delta_{\eta}^{1 / 3}}{\delta_{A, 0}^{2 / 3}}\right)^{2} \frac{B_{0}}{4 \sigma}, \\
\beta_{A}=1-2 \frac{\delta_{\eta}^{2 / 3}}{\delta_{\eta}^{2 / 3}+\delta_{A, 0}^{4 / 3}},
\end{gathered}
$$$$
\delta_{A, 0}=\sqrt{\frac{1+\beta_{A, 0}}{1-\beta_{A, 0}}}, \quad \beta_{A, 0}=\sqrt{\sigma /(1+\sigma)} .
$$

Plasma density at each point is $\rho=\left(U_{A}^{2} / \sigma\right) \rho_{0}$ and magnetic field is $B=\left(U_{A}^{2} / \sigma\right) B_{0}$. We stress that these solutions are exact; no assumptions about the value of the parameter $\sigma$ were made.

In the strongly magnetized limit $\sigma \rightarrow \infty$ we find

$$
\begin{gathered}
\beta=1-\frac{1}{2^{1 / 3} \delta_{\eta}^{4 / 3} \sigma^{2 / 3}}, \\
\gamma=\left(\frac{\sigma \delta_{\eta}^{2}}{2}\right)^{1 / 3}, \\
U_{A}=\left(\frac{\sigma}{2 \delta_{\eta}}\right)^{1 / 3}=\sqrt{\frac{\sigma}{2 \gamma}} .
\end{gathered}
$$

The front of the rarefaction wave is located where $\delta_{\beta}=1$. This gives $\delta_{\eta}=1 / \delta_{A, 0}$,

$$
\eta_{\mathrm{RW}}=-\sqrt{\frac{\sigma}{1+\sigma}} .
$$

Thus, the rarefaction wave propagates into undisturbed plasma with the Alfvén velocity in the unperturbed plasma, $\beta_{A}=-\sqrt{\frac{\sigma}{1+\sigma}}$. 
The vacuum interface corresponds to zero magnetic pressure, where $\delta_{A}=1$ [Eqs. (20)]. This occurs at $\delta_{\eta}=\delta_{\text {vac }}=\delta_{A, 0}^{2}$ (this corresponds to the maximum allowed value of $\delta_{\eta}$ ). Vacuum interface is located at

$$
\eta_{\mathrm{vac}}=2 \frac{\sqrt{\sigma(1+\sigma)}}{(1+2 \sigma)} \approx \begin{cases}1-1 /\left(8 \sigma^{2}\right) & \text { if } \sigma \gg 1 \\ 2 \sqrt{\sigma} & \text { if } \sigma \ll 1 .\end{cases}
$$

The Lorentz factor of the vacuum interface is exactly

$$
\gamma_{\mathrm{vac}}=\frac{1}{\sqrt{1-\eta_{\mathrm{vac}}^{2}}}=1+2 \sigma .
$$

The corresponding four-velocity is

$$
U_{\mathrm{vac}}=\eta_{\mathrm{vac}} \gamma_{\mathrm{vac}}=2 \sqrt{\sigma(1+\sigma)} \approx \begin{cases}2 \sigma & \text { if } \sigma \gg 1 \\ 2 \sqrt{\sigma} & \text { if } \quad \sigma \ll 1 .\end{cases}
$$

Since in the limit $\sigma \ll 1$ the initial Alfvén velocity is $\beta_{A, 0}$ $=\sqrt{\sigma}$, the front expands with $v_{\mathrm{vac}}=2 \beta_{A, 0}$, in agreement with known results for polytropic fluid (expansion into vacuum with velocity $\left.2 c_{s} /(\Gamma-1)[17-19]\right)$.

The flow becomes sonic, $\beta_{A}=\beta$ always at $\eta=0$, and $\delta_{\eta}$ $=1$; this is similar to the general case of nonrelativistic expansion of polytropic gas into vacuum [20] (Sec. 20). At this point the four-velocity of the flow

$$
U(\eta=0) \approx \begin{cases}(\sigma / 2)^{1 / 3} & \text { if } \sigma \gg 1 \\ 2 \sqrt{\sigma} / 3 & \text { if } \sigma \ll 1 .\end{cases}
$$

Equations for forward and backward characteristics are

$$
\begin{gathered}
\left.d_{t} z\right|_{+}=\frac{\beta+\beta_{A}}{1+\beta \beta_{A}}=\frac{\delta_{A, 0}^{8 / 3} \delta_{\eta}^{2 / 3}-1}{1+\delta_{A, 0}^{8 / 3} \delta_{\eta}^{2 / 3}}, \\
\left.d_{t} z\right|_{-}=\frac{\beta-\beta_{A}}{1-\beta \beta_{A}}=\frac{z}{t} .
\end{gathered}
$$

The backward characteristics is a straight line, $z=\eta t$, with $\eta_{\text {rar }}<\eta<\eta_{\text {vac. }}$. Equation for the forward characteristics integrates to give

$$
t=\mathrm{const} \frac{\left(1+\delta_{\eta}^{2}\right)}{\left(\delta_{A, 0}^{8 / 3}-\delta_{\eta}^{4 / 3}\right)^{3 / 2}}, \quad \delta_{\eta}<\delta_{A, 0}^{2} .
$$

For a characteristics that starts at a point $t=t_{0}, \quad z=$ $-t_{0} \sqrt{\sigma /(1+\sigma)}$, this gives

$$
\frac{t}{t_{0}}=\left(\delta_{A, 0}^{2}-1\right) \sqrt{\delta_{A, 0}^{4}-1} \frac{\left(1+\delta_{\eta}^{2}\right)}{\left(\delta_{A, 0}^{8 / 3}-\delta_{\eta}^{4 / 3}\right)^{3 / 2}} .
$$

Thus, the forward characteristics never cross the vacuum interface $\left(\delta_{\eta}=\delta_{A, 0}^{2}\right)$ and becomes parallel to it as $t \rightarrow \infty$.

The flow lines are given by

$$
\frac{d z}{d t}=\beta=\frac{\delta_{A, 0}^{4 / 3} \delta_{\eta}^{4 / 3}-1}{1+\delta_{A, 0}^{4 / 3} \delta_{\eta}^{4 / 3}},
$$

which integrates to give

$$
t=\operatorname{const} \frac{\left(1+\delta_{\eta}^{2}\right)}{\left(\delta_{A, 0}^{8 / 3}-\delta_{\eta}^{4 / 3}\right)^{3}}, \quad \delta_{\eta}<\delta_{A, 0}^{2} .
$$

In particular, for a flow line starts at a point $t=t_{0}, z=$ $-t_{0} \sqrt{\sigma /(1+\sigma)}$, this gives

$$
\frac{t}{t_{0}}=\frac{\left(\delta_{A, 0}^{2}-1\right)^{3}}{1+\delta_{A, 0}^{2}} \frac{\left(1+\delta_{\eta}^{2}\right)}{\left(\delta_{A, 0}^{8 / 3}-\delta_{\eta}^{4 / 3}\right)^{3}} .
$$

Thus, both the characteristics and the flow lines asymptote to $\delta_{\eta} \rightarrow \delta_{A, 0}^{2}$.

For $\sigma \gg 1$ the expansion is relativistic practically in the whole flow. In the bulk, $\gamma \sim \sigma^{1 / 3}$ [e.g., at the sonic point $\eta$ $\left.=0, \gamma=(\sigma / 2)^{1 / 3}\right]$. In a narrow region near $\eta_{\mathrm{vac}}$, with thickness of the order $\Delta \eta \sim 1 / \sigma^{2}$, the Lorentz factor of the flow approaches $\gamma_{\text {vac }}$ (at this $\Delta \eta$ the Lorentz factor reaches half of its maximum values). As the flow expands, the local magnetization, $U_{A}^{2}$, decreases.

The energy flux

$$
T_{0 z}=\gamma^{2}\left(\rho+B^{2}\right) \beta=\frac{\left(\delta_{\eta}^{4 / 3}-\delta_{A, 0}^{8 / 3}\right)^{2}\left(\delta_{\eta}^{8 / 3} \delta_{A, 0}^{8 / 3}-1\right)}{64 \delta_{A, 0}^{4} \delta_{\eta}^{8 / 3} \sigma} \rho_{0}
$$

reaches maximum at $\eta=0$. In the limit $\sigma \gg 1$ it stays nearly constant in between $\eta_{\mathrm{RW}}$ and $\eta_{\text {vac }}$ at a value $T_{0 z} \approx B_{0}^{2} / 4$. The energy density component,

$$
\begin{aligned}
T_{00} & =\gamma^{2}\left(B^{2}+\rho\right)-B^{2} / 2 \\
& =\frac{\left(\delta_{A, 0}^{4 / 3}-\delta_{\eta}^{2 / 3}\right)^{2}\left[\left(\delta_{A, 0}^{4 / 3}+\delta_{\eta}^{2 / 3}\right)^{2}\left(1+\delta_{A, 0}^{8 / 3} \delta_{\eta}^{8 / 3}\right)+8 \delta_{A, 0}^{8 / 3} \delta_{\eta}^{2}\right]}{64 \delta_{A, 0}^{4} \delta_{\eta}^{8 / 3} \sigma} \rho_{0},
\end{aligned}
$$

decreases toward the vacuum interface, remaining nearly constant in between $\eta_{\mathrm{RW}}$ and $\eta_{\mathrm{vac}}$ at the same value $T_{00}$ $\approx B_{0}^{2} / 4$. It may be verified by direct calculations that the total energy in the flow, integral of $T_{00}$ from $\eta_{\mathrm{RW}}$ to $\eta_{\mathrm{vac}}$, equals the total energy in the initial state between $\eta_{\mathrm{RW}}$ and 0 , with the energy density equal to $\rho_{0}(1+\sigma / 2)$.

\section{Moving piston: Expansion into vacuum}

Let us now assume that in the undisturbed plasma, the piston is moving with velocity $\beta_{w}$ toward the external medium. The corresponding relations can be trivially obtained using Riemann invariants and imposing a condition that the piston is moving with velocity $\beta_{w}$ and corresponding Doppler factor $\delta_{w}=\sqrt{\left(1+\beta_{w}\right) /\left(1-\beta_{w}\right)}$. Thus, in the previous relations we need to make a substitution $\delta_{A, 0} \rightarrow \delta_{A, 0} \sqrt{\delta_{w}}$ (this choice assumes that the magnetized medium moves toward the unmagnetized one; in the opposite case, $\delta_{A, 0} \rightarrow \delta_{A, 0} / \sqrt{\delta_{w}}$ ). We then find exact solution of relativistic Riemann problem for expansion into vacuum of cold strongly magnetized plasma with magnetization parameter $\sigma$ and moving with velocity $\beta_{w}$,

$$
\begin{gathered}
\delta_{\beta}=\delta_{\eta}^{2 / 3} \delta_{A, 0}^{2 / 3} \delta_{w}^{1 / 3}, \\
\delta_{A}=\frac{\delta_{A, 0}^{2 / 3} \delta_{w}^{1 / 3}}{\delta_{\eta}^{1 / 3}},
\end{gathered}
$$




$$
\begin{gathered}
\gamma=\frac{1}{2}\left(\delta_{\eta}^{2 / 3} \delta_{A, 0}^{2 / 3} \delta_{w}^{1 / 3}+\frac{1}{\delta_{\eta}^{2 / 3} \delta_{A, 0}^{2 / 3} \delta_{w}^{1 / 3}}\right), \\
\beta=\frac{\delta_{A, 0}^{4 / 3} \delta_{\eta}^{4 / 3} \delta_{w}^{2 / 3}-1}{1+\delta_{A, 0}^{4 / 3} \delta_{\eta}^{4 / 3} \delta_{w}^{2 / 3}} \\
U_{A}=\frac{1}{2}\left(\frac{\delta_{A, 0}^{2 / 3} \delta_{w}^{1 / 3}}{\delta_{\eta}^{1 / 3}}-\frac{\delta_{\eta}^{1 / 3}}{\delta_{A, 0}^{2 / 3} \delta_{w}^{1 / 3}}\right), \\
\beta_{A}=1-2 \frac{\delta_{\eta}^{2 / 3}}{\delta_{\eta}^{2 / 3}+\delta_{A, 0}^{4 / 3} \delta_{w}^{2 / 3}} .
\end{gathered}
$$

(In case of a boost away from the contact, we should substitute $\delta_{w}^{1 / 3} \rightarrow \delta_{w}^{-1 / 3}$, in accordance with the Lorentz transformation of the Doppler factor.)

The vacuum interface is moving with $\delta_{\eta}=\delta_{A, 0}^{2} \delta_{w}$, which in the limit $\sigma, \gamma_{w} \gg 1$ gives $\gamma_{\text {vac }}=2 \gamma_{w}(1+2 \sigma)$.

The front of the rarefaction wave is located at $\delta_{\beta}=\delta_{w}$, which gives

$$
\delta_{\eta}=\frac{\delta_{w}}{\delta_{A, 0}} \approx \frac{\gamma_{w}}{\sqrt{\sigma}} .
$$

The front of the rarefaction wave is stationary (located at $\eta$ $=0, \delta_{\eta}=1$ ) when the flow moves sonically, with the Alfvén velocity of the undisturbed plasma, $\beta_{w}=\beta_{A, 0}$. We stress that these solutions are exact; no assumptions about the value of the parameter $\sigma$ and the velocity $\beta_{w}$ were made.

\section{Reflection of the rarefaction wave from the wall}

Let us now assume that the initial state with $\beta_{w}=0$ occupies a limited region of space $-L<z<0$, with impenetrable wall at $z=-L$. As the rarefaction wave reaches the wall, it will be reflected, creating a secondary RW. The initial RW propagates to the left, while the secondary RW propagates to the right into the region disturbed by the first RW. Let us denote $\beta_{\mathrm{RW}, 2}$ as the velocity of the secondary RW. Its front propagates along the forward characteristics with velocity given in Eq. (29) (Fig. 3).

In this case the expansion is non-self-similar since there is a typical scale in the problem $L$. In addition, even at asymptotically long times after the beginning of the flow, at times $t \gg L / c$, the expansion of a magnetic shell is not self-similar since conservation of mass (and magnetic flux) results in different scaling of magnetic $\left(\propto B^{2}\right)$ and rest mass energy densities.

Still, it might be possible to find exact solution for the expansion of a magnetized layer into plasma following the corresponding hydrodynamic approach [21]. (One can always replace the nonlinear problem of one-dimensional hydrodynamic evolution with a linear equation using [22] transform.) We leave this problem for future; here, we consider the motion of the front of the secondary RW.

Let us discuss the limiting cases of flow evolution long after the reflection of the secondary RW from the wall, $t$ $\gg t_{0}$. In the high magnetization limit, $\delta_{A, 0}=2 \sqrt{\sigma} \gg 1$, and for large Lorentz factors of the secondary $\mathrm{RW}, \beta_{\mathrm{RW}, 2} \sim 1$ $-1 /\left(2 \gamma_{\mathrm{RW}, 2}^{2}\right)$, Eq. (29) gives
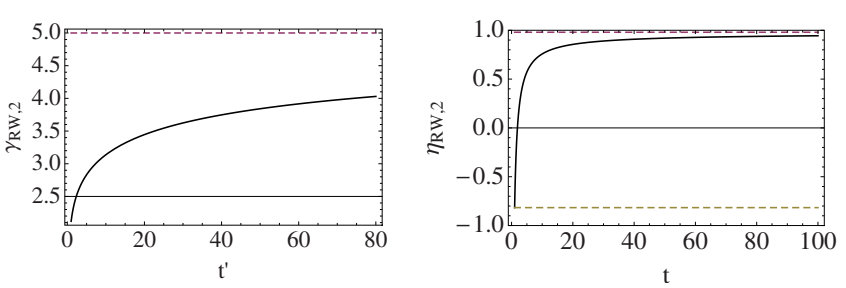

FIG. 3. (Color online) Left panel: Lorentz factor of the secondary rarefaction wave as a function of $t^{\prime}$ time since reflection. The secondary RW propagates in the flow disturbed by the primary RW. In the limit $t^{\prime} \gg 8 \sigma^{2} t_{0}$, the secondary RW reaches the terminal Lorentz factor of the vacuum interface $\gamma_{\mathrm{vac}}=1+2 \sigma$ (dashed line). The plot is for $\sigma=2$. The limiting value of the Lorentz factor is reached very slowly, with $\gamma_{\mathrm{RW}, 2}=\sqrt{2} \sigma^{2 / 3}\left(t / t_{0}\right)^{1 / 6}$ for $t \ll 8 \sigma^{2} t_{0}$. Right panel: motion of the secondary RW in self-similar coordinates $\eta=z / t$. At time $t_{0}=1$, secondary RW is launched from $z=-\sqrt{\sigma /(1+\sigma)} t_{0}$, corresponding to $\eta=-\sqrt{\sigma /(1+\sigma)}$ (lower dashed line) in the forward direction. At $t \rightarrow \infty$ the front of the RW approaches the vacuum interface $\eta=2 \sqrt{\sigma(1+\sigma)} /(1+2 \sigma)$ (upper dashed line).

$$
\frac{1}{2 \gamma_{\mathrm{RW}, 2}^{2}}=\frac{\left[t_{0}+t /\left(2 \gamma_{\mathrm{RW}, 2}^{2}\right)\right]^{1 / 3}}{4 t^{1 / 3} \sigma^{4 / 3}} .
$$

For a long time, when $t \leq 2 t_{0} \gamma_{\mathrm{RW}, 2}^{2}$, the rarefaction accelerates only slowly,

$$
\gamma_{\mathrm{RW}, 2}=\sqrt{2} \sigma^{2 / 3}\left(t / t_{0}\right)^{1 / 6} \quad \text { if } t \leq 8 t_{0} \sigma^{2} .
$$

For $t>2 t_{0} \gamma_{\mathrm{RW}, 2}^{2}$, which using Eq. (38) implies $t>8 t_{0} \sigma^{2}$, the secondary RW reaches the terminal Lorentz factor $\gamma_{\mathrm{RW}, 2}$ $\approx 2 \sigma$, the Lorentz factor of the vacuum interface. In selfsimilar coordinates, the front of the secondary RW moves from $\eta=-\sqrt{\sigma /(1+\sigma)}$ to $\eta=2 \sqrt{\sigma(1+\sigma)} /(1+2 \sigma)$ (Fig. 3). Note that even in the case when the second rarefaction wave approaches the vacuum interface, the expansion is still generically non-self-similar since there are two conserved quantities, energy, and magnetic flux, which scale differently with radius.

The velocity of plasma in front of the reflected rarefaction waves is given in Eq. (30). The flow initially accelerates

$$
\gamma=\left(t / t_{0}\right)^{1 / 3} \sigma^{1 / 3}
$$

and reaches terminal Lorentz factor $\gamma=1+2 \sigma$ at times $t$ $\geq 8 t_{0} \sigma^{2}$ (see also [23]). As the secondary rarefaction wave catches up with the flow, the flow is decelerated. Thus, most of the acceleration occurs in a regime when the flow is causally disconnected from the wall (contrary to the claim in Ref. [23]).

\section{ONE-DIMENSIONAL EXPANSION OF MAGNETIC PISTON INTO PLASMA}

\section{A. Stationary initial conditions}

The solution for the expansion of magnetized plasma into vacuum derived in Sec. II can be used to consider a decay of a contact discontinuity (CD) between magnetized plasma with density $\rho_{0}$ and magnetization parameter $\sigma$ and a cold plasma with density $\rho_{\mathrm{ex}}$. For clarity, we refer below to the 
magnetized component as a piston and the nonmagnetized plasma as the external medium.

For cold external plasma, we expect the formation of four regions: undisturbed external plasma, shocked external plasma, expanding magnetic piston, and undisturbed magnetized plasma. These regions are separated by the forward shock, a contact discontinuity, and an expansion wave. We expect that the dynamics of the system will be self-similar.

The solution for simple waves derived in Sec. II can be used to describe the expansion wave part of the flow. For nonzero external density, the simple waves will terminate at a $\mathrm{CD}$, located at a particular value of the self-similar variable $\eta_{\mathrm{CD}}<\eta_{\mathrm{vac}}$, so that the magnetic pressure of the expansion wave balances the thermal pressure of the shocked medium, which, in turn, depends of the velocity of the CD and external density $\rho_{\mathrm{ex}}$.

Let $\beta_{1}$ and $\beta_{2}$ be the velocities of the unshocked and shocked plasmas in the frame of the forward shock. The shock jump conditions require [24] (assuming $\left.p_{1}=0\right)$

$$
\begin{gathered}
\beta_{1}^{2}=\frac{\epsilon_{2} p_{2}}{\left(\epsilon_{2}-\rho_{\mathrm{ex}}\right)\left(\rho_{\mathrm{ex}}+p_{2}\right)}, \\
\beta_{2}^{2}=\frac{p_{2}\left(p_{2}+\rho_{\mathrm{ex}}\right)}{\epsilon_{2}\left(\epsilon_{2}-\rho_{\mathrm{ex}}\right)},
\end{gathered}
$$

where $\epsilon_{2}$ and $p_{2}$ are energy density and pressure in the shocked medium and we assumed that the external medium is cold, $p_{\text {ex }}=0, \epsilon_{\text {ex }}=\rho_{\text {ex }}$.

In the frame of the $\mathrm{CD}(\mathrm{CD}$ is stationary with respect to downstream plasma), the shock has velocity $v_{2}$, while the incoming flow has velocity $v_{12}$. In the observer' $\mathrm{s}$ frame, the CDs have velocity $v_{\mathrm{CD}}$, while the shock has

$$
\beta_{\mathrm{FS}}=\frac{\beta_{\mathrm{CD}}+\beta_{2}}{1+\beta_{\mathrm{CD}} \beta_{2}} .
$$

Since the upstream plasma is at rest in the observer's frame

$$
\beta_{\mathrm{CD}}=v_{12} \text {. }
$$

Equations (42), an equation of state $\epsilon_{2}\left(p_{2}\right)$, a condition of force balance on $\mathrm{CD}$,

$$
p_{2}=B^{2} / 2=U_{A}\left(\eta=\eta_{\mathrm{CD}}\right)^{4} \rho_{0} /(2 \sigma),
$$

and shock jump conditions [9],

$$
\begin{aligned}
\left(\frac{\mathcal{E}_{1}+\rho_{1}+p_{1}}{\rho_{\mathrm{ex}}}\right)^{2}= & \left(\frac{\mathcal{E}_{2}+\rho_{2}+p_{2}}{\rho_{2}}\right)^{2} \\
& -\left(p_{2}-p_{1}\right)\left(\frac{\mathcal{E}_{1}+\rho_{1}+p_{1}}{\rho_{\mathrm{ex}}^{2}}+\frac{\mathcal{E}_{2}+\rho_{2}+p_{2}}{\rho_{2}^{2}}\right),
\end{aligned}
$$

constitute an equation on $\eta_{\mathrm{CD}}$, the location of the contact discontinuity. [In Eq. (44) indices 1 and 2 refer to unshocked and shocked external media correspondingly, $\rho_{1} \equiv \rho_{\text {ex }}$.]

For cold external plasma, $p_{1}=0$ and assuming adiabatic index of $\Gamma=4 / 3$ (so that $\mathcal{E}_{2}=3 p_{2}+\rho_{2}$ ), the shock jump condition gives
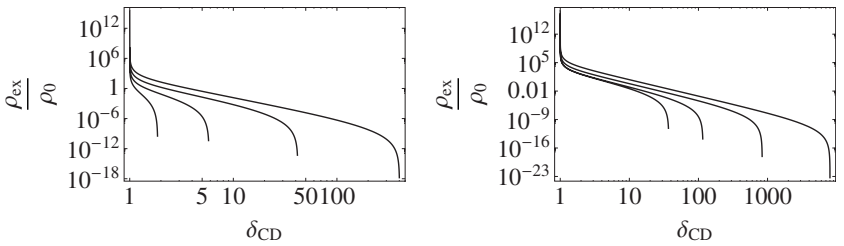

FIG. 4. Ratio of external density to the piston density $\rho_{\mathrm{ex}} / \rho_{0}$ as a function of the Doppler factor of the contact discontinuity $\delta_{\mathrm{CD}}$ for one-dimensional expansion of strongly magnetized plasma with magnetization $\sigma, \sigma=100,10,1,0.1$, top to bottom curves. Left panel: stationary initial conditions. Right panel: piston moving with $\gamma_{w}=10$. Maximum values of $\delta_{\mathrm{CD}}=\delta_{A, 0}^{2}$ (left panel) and $\delta_{\mathrm{CD}}$ $=\delta_{A, 0}^{2} \delta_{w}$ (right panel) correspond to zero external density, a vacuum interface.

$$
\rho_{2}=\frac{1}{2}\left[7 \rho_{1}+\sqrt{\rho_{\mathrm{ex}}\left(48 p_{2}+49 \rho_{\mathrm{ex}}\right)}\right] .
$$

Using continuity equations [Eqs. (40)-(42)], solutions for the expansion flow given in Eqs. (20), and shock jump condition (44) we find

$$
\frac{\rho_{\mathrm{ex}}}{\rho_{0}}=-\frac{3\left(\delta_{A, 0}^{2}-\delta_{\beta, \mathrm{CD}}\right)^{4}}{16 \sigma \delta_{A, 0}^{4}\left(1-\delta_{\beta, \mathrm{CD}}^{2}\right)\left(2+3 \delta_{\beta, \mathrm{CD}}+2 \delta_{\beta, \mathrm{CD}}^{2}\right)} .
$$

Equation (46) determines the Doppler factor of the contact discontinuity for plasma obeying equation of state with polytropic index $\Gamma=2$, having initial Alfvén Doppler factor $\delta_{A, 0}$ expanding into a medium with density $\rho_{\text {ex }}$; the external medium is unmagnetized and obeys an equation of state with polytropic index $\Gamma=4 / 3$. Equation (46) is relativistically exact; no assumption about values of magnetization or external density were made (see Fig. 4). Note that it correctly reproduces expansion into vacuum, $\rho_{\mathrm{ex}}=0, \delta_{\mathrm{vac}}=\delta_{A, 0}^{2}$. Thus, shock jump condition (44) is also applicable to the plasmavacuum interface.

In the limit of relativistically strong shock, when the postshock pressure is much larger than the postshock density, relation (46) gives

$$
\gamma_{\mathrm{CD}}=\left(\frac{3}{32} \frac{\rho_{0}}{\rho_{\mathrm{ex}}} \sigma\right)^{1 / 4}=\left(\frac{3}{32} \frac{B_{0}^{2}}{\rho_{\mathrm{ex}}}\right)^{1 / 4} .
$$

In this limit the velocity of the forward shock in the observer frame, $\beta_{\mathrm{FS}}=\left(\beta_{\mathrm{CD}}+\beta_{2}\right) /\left(1+\beta_{\mathrm{CD}} \beta_{2}\right)$, is

$$
\gamma_{\mathrm{FS}}=\sqrt{2} \gamma_{\mathrm{CD}}=\left(\frac{3}{8} \frac{\rho_{0}}{\rho_{\mathrm{ex}}} \sigma\right)^{1 / 4}=\left(\frac{3}{8} \frac{B_{0}^{2}}{\rho_{\mathrm{ex}}}\right)^{1 / 4} .
$$

Heuristically, expressions (47) and (48) can be obtained as a pressure balance at time $t=0+$ when the contact instantaneously reaches a Lorentz factor $\gamma_{\mathrm{CD}}$; the magnetic pressure in the plasma frame then is $\sim B^{2} / \gamma_{\mathrm{CD}}^{2}$ and the kinetic pressure of the shocked external medium is $\sim \rho_{\mathrm{ex}} \gamma_{\mathrm{CD}}^{2}$.

Note that in the strong forward shock (FS) limit, the Lorentz factor of the CD, and of the forward shock, Eqs. (47) and (48) are independent of $\sigma$, the composition of the driver. Only the value of total pressure in the piston matters $\left(\propto B_{0}^{2}\right.$ in our case). Composition of the driver becomes important only for weak nonrelativistic FS when the Lorentz factor of CD 
(47) approaches the limit of expansion into vacuum, $\gamma_{\mathrm{vac}}$ $=1+2 \sigma$. This occurs for

$$
\rho_{\mathrm{ex}}<\frac{3}{256} \frac{1}{\sigma^{3}} \rho_{0} .
$$

In this limit of small external density, $\rho_{\mathrm{ex}} \rightarrow 0$, we find

$$
\gamma_{\mathrm{CD}}=1+\left[2-\left(\frac{14336}{3} \frac{\rho_{\mathrm{ex}}}{\rho_{0}}\right)^{1 / 4}\right] \sigma \text {. }
$$

\section{B. Expansion into medium: Moving piston}

For moving piston, the system becomes somewhat more complicated. Similarly to the stationary case, we expect the formation of four regions: undisturbed external plasma, shocked external plasma, and undisturbed magnetized plasma; however, the expanding magnetic piston may now turn into region of shocked piston material. These regions are separated by the forward shock, a contact discontinuity, and an expansion wave or the reverse shock front.

The cases of the expansion wave and the reverse shock have somewhat different dynamics. In case of the expansion wave, the solution for simple waves derived in Sec. II can be used to describe the expansion wave part of the flow. Alternatively, if reverse shock (RS) shock is launched, which heats the piston material, the pressure on $\mathrm{CD}$ is determined by the sum of the magnetic pressure of magnetic field compressed at the RS and the kinetic pressure of shocked particles.

\section{Forward shock and rarefaction wave}

In case of magnetic piston moving with initial Doppler factor $\delta_{w}$ into cold medium with density $\rho_{\mathrm{ex}}$, the CD is moving with the Doppler factor $\delta_{\beta, \mathrm{CD}, w}$ determined from

$$
\frac{\rho_{\mathrm{ex}}}{\rho_{0}}=-\frac{3\left(\delta_{A, 0}^{2} \delta_{w}-\delta_{\beta, \mathrm{CD}, w}\right)^{4}}{16 \sigma \delta_{A, 0}^{4} \delta_{w}^{2}\left(1-\delta_{\beta, \mathrm{CD}, w}^{2}\right)\left(2+3 \delta_{\beta, \mathrm{CD}, w}+2 \delta_{\beta, \mathrm{CD}, w}^{2}\right)} .
$$

For strong forward shocks, the Lorentz factors of the CD and the FS are

$$
\begin{aligned}
\gamma_{\mathrm{CD}, w} & =\left(\frac{3 B_{0}^{2} \gamma_{w}^{2}}{8 \rho_{\mathrm{ex}}}\right)^{1 / 4}, \\
\gamma_{\mathrm{FS}, w} & =\left(\frac{3 B_{0}^{2} \gamma_{w}^{2}}{2 \rho_{\mathrm{ex}}}\right)^{1 / 4}
\end{aligned}
$$

(see Fig. 4).

Composition of the driver becomes important only for weak FS when the Lorentz factor of CD (47) approaches the limit of expansion into vacuum, $\Gamma=2 \gamma_{w}(1+2 \sigma)$. This occurs for

$$
\rho_{\mathrm{ex}}<\frac{3}{1024} \frac{1}{\gamma_{w}^{2} \sigma^{3}} \rho_{0} .
$$

The front of the rarefaction wave is stationary (located at $\eta$ $=0, \delta_{\eta}=1$ ) when the flow moves sonically, with the Alfvén velocity of the undisturbed plasma, $\beta_{w}=\beta_{A, 0}$. For higher $\beta_{w}$ the front of the RW is advected forward.

\section{Formation of a RS: Supersonic motion of eject in the CD frame}

For sufficiently high velocity $\beta_{w}$, the location of the RW [Eq. (22)] coincides with the location of the FS, $\delta_{\eta, \mathrm{FS}}$ $\approx 2 \gamma_{\mathrm{CD}}$. This occurs for

$$
\gamma_{w}>2 \gamma_{\mathrm{CD}} \sqrt{\sigma}
$$

that is, when the wind velocity in the frame of the $\mathrm{CD}$ is supersonic, an obvious condition. In terms of initial magnetization and the ratio of densities, the RS forms when

$$
\gamma_{w}>\gamma_{w, \text { crit }}=\sqrt{\frac{3}{8} \frac{\rho_{0}}{\rho_{\mathrm{ex}}} \sigma \delta_{A, 0}^{2}}=\left\{\begin{array}{cl}
\sqrt{\frac{3}{8} \frac{\rho_{0}}{\rho_{\mathrm{ex}}}}, & \sigma \ll 1 \\
\sqrt{6 \frac{\rho_{0}}{\rho_{\mathrm{ex}}} \sigma^{3 / 2}}, & \sigma \gg 1 .
\end{array}\right.
$$

Relation (55) assumes strong FS and high initial Lorentz factor $\gamma_{w} \gg 1$.

\section{Formation of a RS: Subsonic motion of piston in the CD frame}

When motion of the piston in the frame of the CD is subsonic, yet the piston is moving faster than the $\mathrm{CD}, \gamma_{\mathrm{CD}}$ $<\gamma_{w}<2 \gamma_{\mathrm{CD}} \sqrt{\sigma}$, the flow is decelerated by a compression wave. For one-dimensional motion the compression waves are unstable to formation of shocks [24], so that the reverse shock will form for $\gamma_{w}>\gamma_{\mathrm{CD}}$ and not at condition (54). As long as $\gamma_{w}<2 \gamma_{\mathrm{CD}} \sqrt{\sigma}$, the reverse shock is weak; in this range the RS may not form if a more complicated flow patters are allowed. For $\gamma_{w}>2 \gamma_{\mathrm{CD}} \sqrt{\sigma}$, RS becomes strong. (We define strong shocks as the shocks in which the upstream four-velocity in the frame of the shock is much larger than the upstream Alfvén velocity.)

\section{Reverse shock}

For sufficiently fast initial velocity, satisfying $\gamma_{w} \gg \gamma_{w, c r i t}$, highly magnetized, $\sigma \gg 1$, reverse shock forms. In this section we consider the dynamics of double-shock structures. For convenience, we will make two approximations; first, we assume that the forward shock is strong and unmagnetized; second, we assume that the reverse shock is strong as well, $\gamma_{w} \gg \gamma_{w, c r i t}$. We have to solve simultaneously for two shock jump condition and flow continuity at the CD.

In the frame of the $\mathrm{CD}$, the $\mathrm{FS}$ is moving with $\beta_{\mathrm{FS}}^{\prime}=1 / 3$, while reverse shock is moving with [3]

$$
\beta_{\mathrm{RS}}^{\prime, 2}=\frac{1+10 \sigma+8 \sigma^{2}+(1+2 \sigma) \sqrt{1+16 \sigma+16 \sigma^{2}}}{17+26 \sigma+8 \sigma^{2}+(1+2 \sigma) \sqrt{1+16 \sigma+16 \sigma^{2}}} .
$$

In the frame of the shock, the postshock kinetic pressures are [3] 


$$
\frac{p_{2, k i n}}{\rho_{1} u_{1}^{2}}=\frac{\sigma\left(1-\frac{\gamma_{2}}{u_{2}}\right)+1}{4 u_{2} \gamma_{2}}
$$

while magnetic field satisfies

$$
B_{1} \gamma_{1} \beta_{1}=B_{2} \gamma_{2} \beta_{2}
$$

where indices 1 and 2 refer to quantities measured in the upstream and downstream in the frame of the shock.
If the $\mathrm{CD}$ is moving with velocity $\beta_{\mathrm{CD}}$ and the initial velocity is $\beta_{w}$, the velocity of the incoming plasma in the frame of the RS is

$$
v_{1, \mathrm{RS}}^{\prime}=\frac{\left(1-\beta_{\mathrm{CD}} \beta_{w}\right) \beta_{\mathrm{RS}}^{\prime}+\beta_{w}-\beta_{\mathrm{CD}}}{1-\beta_{\mathrm{CD}}\left(\beta_{w}+\beta_{\mathrm{RS}}^{\prime}\right)+\beta_{w} \beta_{\mathrm{RS}}^{\prime}} .
$$

Using the above relations, the pressure behind the FS is

$$
p_{2, \mathrm{FS}}=\frac{\left(1+3 \beta_{\mathrm{CD}}\right)^{2}}{12\left(1-\beta_{\mathrm{CD}}^{2}\right)} \rho_{\mathrm{ex}} \approx \frac{4}{3} \gamma_{\mathrm{CD}}^{2} \rho_{\mathrm{ex}} .
$$

Kinetic pressure behind the RS is

$$
p_{2, k i n, \mathrm{RS}}=\frac{\gamma_{\mathrm{CD}}^{2} \gamma_{w}^{2}\left(\sigma \beta_{\mathrm{RS}}+\beta_{\mathrm{RS}}-\sigma\right)\left[-\beta_{\mathrm{CD}}\left(\beta_{\mathrm{RS}} \beta_{w}+1\right)+\beta_{\mathrm{RS}}+\beta_{w}\right]^{2}}{4 \beta_{\mathrm{RS}}^{2}} \rho_{0} \approx \frac{1}{8} \frac{\gamma_{w}^{2}}{\gamma_{\mathrm{CD}}^{2}} \rho_{0} \quad \text { if } \sigma \gg 1 .
$$

Magnetic pressure is

$$
\begin{aligned}
p_{2, \text { mag }, \mathrm{RS}} & =\frac{1}{2} \gamma_{\mathrm{CD}}^{2} \gamma_{w}^{2}\left[-\beta_{\mathrm{CD}}\left(\beta_{\mathrm{RS}}+\beta_{w}\right)+\beta_{\mathrm{RS}} \beta_{w}+1\right]^{2} \\
& \approx \frac{1}{2} \frac{\gamma_{w}^{2}}{\gamma_{\mathrm{CD}}^{2}} B_{0}^{2} \quad \text { if } \sigma \gg 1 .
\end{aligned}
$$

Balancing pressures (60) and (62), we find

$$
\gamma_{\mathrm{CD}}=\left(\frac{3}{32}(1+4 \sigma) \gamma_{w}^{2} \frac{\rho_{0}}{\rho_{\mathrm{ex}}}\right)^{1 / 4} \approx\left(\frac{3}{8} \gamma_{w}^{2} \frac{B_{0}^{2}}{\rho_{\mathrm{ex}}}\right)^{1 / 4} \text { if } \sigma \gg 1 \text {. }
$$

The Lorentz factor of the CD in case of reverse shock coincides with the one obtained in case of rarefaction wave [Eqs. (52)]. Thus, the forward shock is not influenced by the rarefaction wave-shock wave transition. We stress, again, that in the strong shock limit and for $\sigma \gg 1$, the motion of the CD and the forward shock are independent of the composition of the piston (its density $\rho_{0}$ ).

If we express $\gamma_{\mathrm{CD}}$ as a function of the total luminosity $L / S=\gamma_{w}^{2}\left(B_{0}^{2}+\rho_{0}\right)$ (assuming $\left.\beta_{w} \sim 1\right)$, where $S$ is the cross section of the flow, we find

$$
\gamma_{\mathrm{CD}}=\left(\frac{3}{32} \frac{L}{S \rho_{1}} \frac{1+4 \sigma}{1+\sigma}\right)^{1 / 4}
$$

It is only weakly dependent on $\sigma$.

The RS is stationary in the observer frame when $\beta_{\mathrm{CD}}$ $=\beta_{\mathrm{RS}}^{\prime}$, which for highly magnetized medium $\sigma \gg 1$ (below, in this section, all relations are given for $\sigma \gg 1$ ) gives

$$
\gamma_{w}=\sqrt{\frac{8}{3} \frac{\rho_{\mathrm{ex}}}{\rho_{0}} \sigma}
$$

In this case $\gamma_{\mathrm{CD}}=\sigma^{1 / 2}$, which can be understood as the RS front is receding at almost with $u_{A}=\sigma^{1 / 2}$ in the CD frame. For higher $\gamma_{w}$ the RS is advected toward the interface, while for smaller $\gamma_{w}$ it propagates in the opposite direction. Also, when $\gamma_{\mathrm{CD}}=\gamma_{w}, \gamma_{w}=\sqrt{(3 / 8)\left(\rho_{0} / \rho_{\mathrm{ex}}\right) \sigma}$, there is no reverse shock or rarefaction wave; the motion of the wind matches exactly the motion of the $\mathrm{CD}$.

For values of $\gamma_{w}$ sufficiently different from Eq. (65), the Lorentz factor of the reverse shock in the frame of stationary external medium is then

$$
\gamma_{\mathrm{RS}}=\frac{1}{2}\left(\frac{\sqrt{\sigma}}{\gamma_{\mathrm{CD}}}+\frac{\gamma_{\mathrm{CD}}}{\sqrt{\sigma}}\right)= \begin{cases}\left(\frac{3}{128}\right)^{1 / 4} \frac{\sqrt{\gamma_{w}}}{\sigma^{1 / 4}}\left(\frac{\rho_{0}}{\rho_{\mathrm{ex}}}\right)^{1 / 4} & \text { if } \gamma_{\mathrm{CD}} \gg \sqrt{\sigma}, \text { RS moving forward } \\ \frac{\sigma^{1 / 4}}{6^{1 / 4} \sqrt{\gamma_{w}}}\left(\frac{\rho_{\mathrm{ex}}}{\rho_{0}}\right)^{1 / 4} & \text { if } \gamma_{\mathrm{CD}} \ll \sqrt{\sigma}, \text { RS moving backward. }\end{cases}
$$




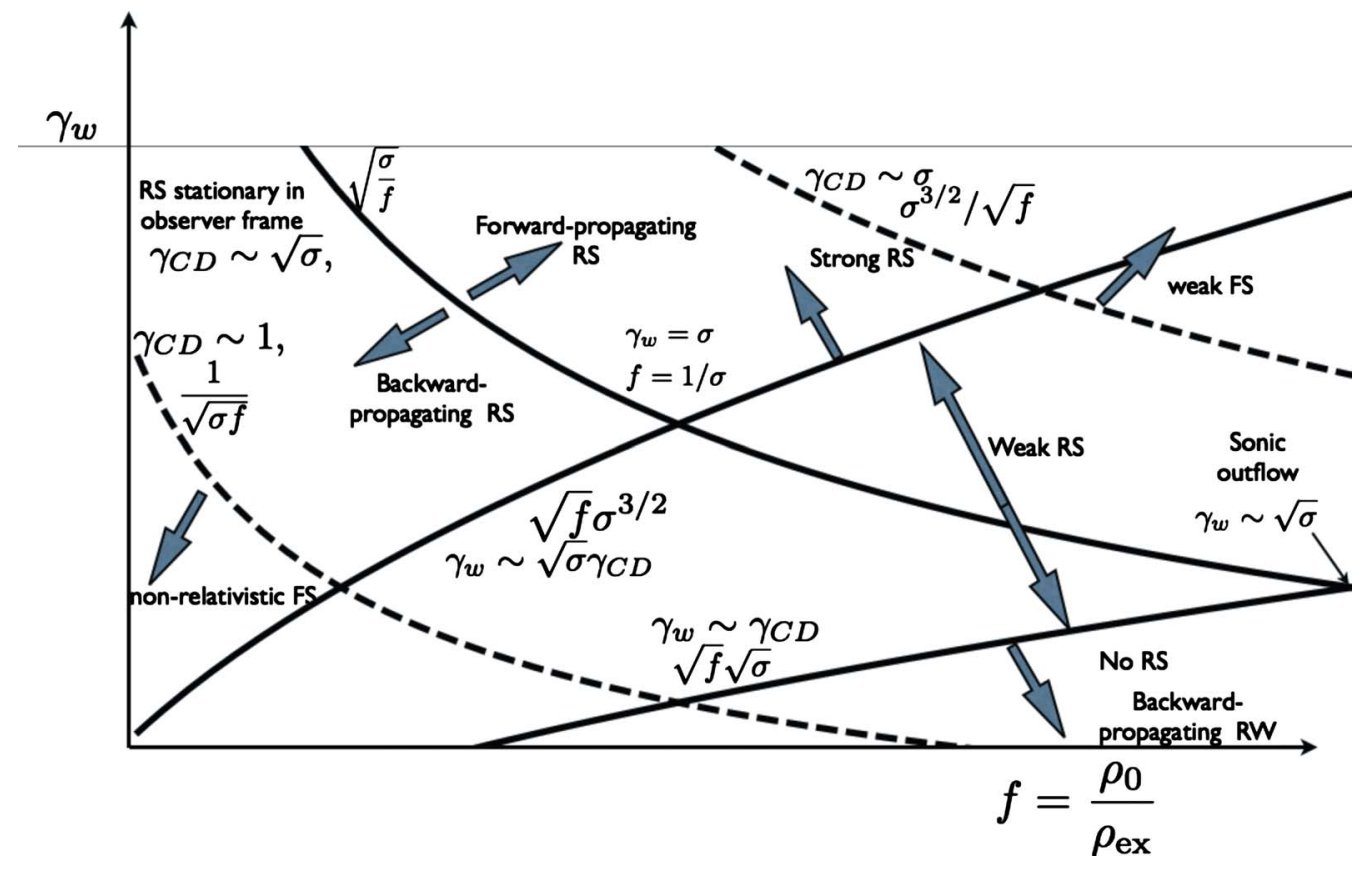

FIG. 5. (Color online) Cartoon of the flow dynamics as a function of two parameters, $\gamma_{w}$, the initial Lorentz factor for the piston and the ratio of piston density to external density $f=\rho_{0} / \rho_{\mathrm{ex}}$. High magnetization is assumed, $\sigma \gg 1$. Solid lines are related to the reverse shock; dashed lines are related to the forward shock. The RS form for $\gamma_{w}>\gamma_{\mathrm{CD}}$, which is $\sqrt{f} \sqrt{\sigma}$ line. Below this line rarefaction wave propagates into piston. The RS shock is weak for $\gamma_{\mathrm{CD}}<\gamma_{w}<2 \gamma_{\mathrm{CD}} \sqrt{\sigma}$; the latter corresponds to $\sqrt{f} \sigma^{3 / 2}$ line. In the frame of the undisturbed plasma the reverse shock propagates in the forward direction for $\gamma_{\mathrm{CD}}>\sqrt{\sigma}$, which corresponds to $\gamma_{w}>\sqrt{f / \sigma}$. The forward shock becomes nonrelativistic for $\gamma_{\mathrm{CD}} \sim 1$, which is the $1 / \sqrt{\sigma f}$ curve. The forward shock becomes weak (so that the postshock temperature is nonrelativistic) for $\gamma_{\mathrm{CD}}$ $\sim \sigma$, which is $\sigma^{3 / 2} / \sqrt{f}$ line. For weak FS, the expansion proceeds nearly as into vacuum. (Since relativistic motion is assumed, the lowest part of the plot, below the intersection of lines $\gamma_{\mathrm{CD}}=1$ and $\gamma_{w}=\gamma_{\mathrm{CD}}$, is outside of the applicability region.)

Recall that relation (66) is applicable only if condition (55) is satisfied.

\section{Upshot: Expansion of magnetized plasma into medium}

In Fig. 5 we qualitatively outline the dynamics of the flow expansion in a medium. As one can see, the dynamics is rich in detail and is much more complicated than for pure hydrodynamical expansion. Qualitatively, as the Lorentz factor of the piston $\gamma_{w}$ increases for given density ration $f$, the rarefaction wave turns first into a weak RS and then into a strong RS. (In non-one-dimensional flows weak reverse shocks can be avoided.) For extremely low external density, the expansion proceeds similarly to the case of expansion into vacuum. As the external density increases, first, the forward shock becomes strong. For higher $\rho_{\mathrm{ex}}$ RS becomes backward propagating.

\section{THREE-DIMENSIONAL EXPANSION INTO VACUUM}

In case of three-dimensional (3D) expansion of plasma carrying toroidal field, we expect that the flow will not be purely radial, as magnetic hoop stresses and magnetic pressure will, generally, induce lateral motion (in $\theta$ direction) in addition to radial expansion. Bur for strongly relativistic motion, corresponding to high magnetization parameter, the $\theta$ component of the velocity will be much smaller by a factor $\sim \gamma^{2}$ than the radial component, so that the motion will be approximately conical, depending only on time and radial coordinate.

For the three-dimensional expansion, the governing equations are generically non-self-similar: there are two conserved quantities, energy and magnetic flux, which scale differently with radius. Self-similarity can still be achieved in a narrow region, e.g., near the surface of the bubble, where different radial scalings of energy and magnetic flux can be neglected.

Similarly to the $1 \mathrm{D}$ case, in case of three-dimensional expansion of magnetized plasma into vacuum, the expansion front reaches terminal velocity immediately and coasts with constant velocity later. The constant terminal velocity in three dimensions is the same as in one dimension-the vacuum interface always propagates with the terminal velocity $\gamma=1+2 \sigma$, independent of the geometry (cf. [18]).

We expect that in the highly relativistic limit, the structure of the outflow will resemble a relativistic shock wave, where parameters change on a scale $\sim \gamma_{\text {vac }}^{2}$ smaller than the overall size of the out flow. It is within this narrow region [and even smaller layer near the vacuum interface; see Eqs. (72) and (73)] that the self-similar solution derived below is applicable. (Greenspan and Butler [18] did consider self-similar $3 \mathrm{D}$ expansion for times much smaller than $r_{0} / c$, while astrophysical applications require times much larger than $r_{0} / c$.)

For conical expansion of plasma carrying toroidal magnetic field, the conservation laws become 


$$
\begin{gathered}
\partial_{t}(\gamma \rho)+\frac{1}{r^{2}} \partial_{r}\left(r^{2} \gamma \beta \rho\right)=0, \\
\partial_{t}(\gamma B)+\frac{1}{r} \partial_{r}(r \gamma \beta B)=0, \\
\partial_{t} T_{00}+\frac{1}{r^{2}} \partial_{r}\left(r^{2} T_{0 r}\right)=0, \\
\partial_{t} T_{0 r}+\frac{1}{r^{2}} \partial_{r}\left(r^{2} T_{r r}\right)=0,
\end{gathered}
$$

where we assumed a constant fixed polar angle $\theta$ and neglected terms involving $\theta$ dependence.

In three dimensions, the continuity and induction equations imply $B=\left(\rho r B_{0}\right) /\left(\rho_{0} r_{0}\right)$, where $r_{0}$ is an initial radius of the magnetized cavity. This scaling implies that for an outflow with a fixed energy, which starts with a finite region of nonzero magnetic field, one cannot assume constant magnetization since $\sigma \propto \rho r^{2}$. Then, the outflow dynamics is not selfsimilar; it depends on the initial $\sigma(r)$. Self-similar solutions exist only very close to the edge of the expanding bubble; we derive them next.

We assume that at the beginning of the expansion, close to the leading front, the plasma parameters (density and magnetic field) do not vary considerably. Eliminating density in favor of the Alfvén four-velocity $\rho=U_{A}^{2} \rho_{0} / \sigma\left(r_{0} / r\right)^{2}$, the condition that the magnetic and rest mass energy densities scale similarly with $r$ requires $U_{A}=g(\chi) / r$. At the vacuum interface the boundary condition is $g=0$. Following [25], we introduce self-similar variable,

$$
\chi=\left(1+2 \gamma_{\mathrm{vac}}^{2}\right)(1-r / t), \quad \chi>1,
$$

and parametrize $\gamma=\gamma_{\mathrm{vac}} f$. (Note that $\gamma_{\mathrm{vac}}$ is a constant.) In the leading orders in $1 / \gamma_{\mathrm{vac}}$ we find

$$
\begin{gathered}
\chi f g^{2}\left(1+g^{2}\right) f^{\prime}-g\left[1-\chi f^{2}\left(1+2 g^{2}\right)\right] g^{\prime}=0, \\
\left(1+\chi f^{2}\right) g f^{\prime}-2 f\left(1-\chi f^{2}\right) g^{\prime}=0 .
\end{gathered}
$$

The solutions satisfying boundary conditions $f(1)=1$ and $g(1)=0$ are

$$
\begin{gathered}
f=\frac{1}{\chi^{1 / 3}}, \\
g=\frac{\chi^{1 / 6}-\chi^{-1 / 6}}{2} .
\end{gathered}
$$

Energy densities per unit range of $\chi$ are given by

$$
\begin{gathered}
U_{B} d \chi=\left(1+\beta^{2}\right) / 2 \gamma^{2} B^{2} \propto\left(1-\chi^{-1 / 3}\right)^{4} d \chi, \\
U_{p} d \chi=\gamma^{2} \rho \propto\left(\chi^{1 / 3}-1\right)^{2} / \chi d \chi .
\end{gathered}
$$

These relations are applicable in the limit $\chi \rightarrow 1$. To estimate the region of applicability, we equate the total energy in the initial bubble, $E \sim B_{0}^{2} r_{0}^{3}$, to the energy within the narrow layer near the surface. We integrate $U_{B}+U_{p}$ from $\eta=1$ to $\eta=1$ $+\Delta \eta$ to determine $\Delta \eta: E \sim B_{0}^{2} r_{0}^{2} t(\Delta \eta)^{3} / \sigma_{0}^{2}$. Equating the two, we find $\Delta \eta$ as the width of the self-similar region in coordinate $\eta$,

$$
\Delta \eta=\left(\frac{r_{0}}{t} \sigma_{0}^{2}\right)^{1 / 3} .
$$

In physical coordinates,

$$
\Delta r=\Delta \eta \frac{t}{2 \Gamma_{0}^{2}}=\left(\frac{r_{0} t^{2}}{8 \sigma_{0}^{4}}\right)^{1 / 3},
$$

where we used $\Gamma_{0} \sim 2 \sigma$.

In conclusion of this chapter, we first reiterate that the terminal velocity of expansion into vacuum in $3 \mathrm{D}$ case is the same as in one dimension: the terminal Lorentz factor is $\gamma$ $=1+2 \sigma$ (if starting at rest). The self-similar structure of the resulting bubble is applicable only in a narrow layer near the expanding vacuum interface; in most of the volume the field and velocity structure do depend on the particular initial conditions in the bubble.

\section{DISCUSSION}

In this paper we found exact explicit solutions for onedimensional relativistic expansion of polytropic fluid into vacuum and into plasma. In particular, we discussed an astrophysical important case of strongly magnetized outflows; in this case especially simple analytical solutions can be obtained. We found exact solutions for one-dimensional expansion of magnetized plasma into vacuum and into the cold medium both for stationary initial conditions and for a piston moving toward the interface. We found exact relations, applicable for arbitrary magnetization, relativistic motion, and external densities. These results can be used for benchmark estimates of the overall dynamical behavior for the numerical simulations of relativistic plasmas, e.g., in heavy-ion collisions and in strongly magnetized outflows in particular.

\section{ACKNOWLEDGMENTS}

I am greatly thankful to Dimitros Gianios, Sergey Komisarov, and Alexandre Tchekhovskoy.
[1] J. M. Martí and E. Müller, Living Rev. Relativ. 6, 7 (2003).

[2] I. Bouras, E. Molnár, H. Niemi, Z. Xu, A. El, O. Fochler, C. Greiner, and D. H. Rischke, Phys. Rev. Lett. 103, 032301 (2009).
[3] C. F. Kennel and F. V. Coroniti, Astrophys. J. 283, 694 (1984).

[4] J. H. Krolik, Active Galactic Nuclei: From the Central Black Hole to the Galactic Environment (Princeton University Press, Princeton, NJ, 1999). 
[5] T. Piran, Phys. Rep. 314, 575 (1999).

[6] E. F. Toro, Riemann Solvers and Numerical Methods for Fluid Dynamics: A Practical Introduction (Springer, NY, 1999).

[7] J. M. Marti and E. Muller, J. Fluid Mech. 258, 317 (1994).

[8] R. Romero, J. M. Martí, J. A. Pons, J. M. Ibáñez, and J. A. Miralles, J. Fluid Mech. 544, 323 (2005).

[9] A. H. Taub, Phys. Rev. 74, 328 (1948).

[10] M. H. Johnson and C. F. McKee, Phys. Rev. D 3, 858 (1971).

[11] M. Lyutikov, New J. Phys. 8, 119 (2006).

[12] M. Lyutikov, Gamma Ray Bursts: Back to the Blackboard (unpublished).

[13] R. D. Blandford and R. L. Znajek, Mon. Not. R. Astron. Soc. 179, 433 (1977).

[14] S. S. Komissarov, Mon. Not. R. Astron. Soc. 359, 801 (2005).

[15] S. S. Komissarov, Mon. Not. R. Astron. Soc. 303, 343 (1999).

[16] L. D. Landau and E. M. Lifshitz, Electrodynamics of Continuous Media (Energy Conversion Management, NY, 1960).
[17] R. Courant and K. O. Friedrichs, Supersonic Flow and Shock Waves (Springer, NY, 1948).

[18] H. P. Greenspan and D. S. Butler, J. Fluid Mech. 13, 101 (1962).

[19] Y. B. Zeldovich and Y. P. Raizer, Physics of Shock Waves (Dover Publications, Inc., New York, 2003).

[20] K. Stanyukovich, Non-Stationary Motion in Continuous Medium (Russian) (State Publishing, Moscow, 1955).

[21] S. Z. Belen'kij and L. D. Landau, Nuovo Cimento, Suppl. 3, 15 (1956).

[22] I. M. Khalatnikov, Zh. Eksp. Teor. Fiz. 26, 529 (1954).

[23] J. Granot, S. Komissarov, and A. Spitkovsky, e-print arXiv:1004.0959, Mon. Not. R. Astron. Soc. (to be published).

[24] L. D. Landau and E. M. Lifshitz, Course of Theoretical Physics (Oxford Press, United Kingdom, 1959).

[25] R. D. Blandford and C. F. McKee, Phys. Fluids 19, 1130 (1976). 